\title{
A Systematic Review of Nonpharmacological Interventions for Moderate to Severe Dementia: A Study Protocol for a Systematic Review and Meta-Analysis
}

\author{
Riyoung $\mathrm{Na}^{1}$, You Joung $\mathrm{Kim}^{1}$, Kiwon Kim${ }^{1,4}$, and Ki Woong Kim, ${ }^{1,2,3,4}$ \\ ${ }^{1}$ National Institute of Dementia, Seongnam, Republic of Korea \\ ${ }^{2}$ Department of Psychiatry, Seoul National University College of Medicine, Seoul, Republic of Korea \\ ${ }^{3}$ Department of Brain and Cognitive Science, Seoul National University College of Natural Sciences, Seoul, Republic of Korea \\ ${ }^{4}$ Department of Neuropsychiatry, Seoul National University Bundang Hospital, Seongnam, Republic of Korea
}

\begin{abstract}
The study is designed as a systematic review on nonpharmacological interventions for patients with moderate to severe dementia. This review will be conducted in accordance with the Cochrane Handbook for Systematic Reviews of Interventions. The following databases will be searched: Cochrane CENTRAL, MEDLINE, EMBASE, CINAHL, PsycINFO, KoreaMED, KMbase, and KISS. The primary outcome will include the effect of the interventions on activities of daily living and behavioral and psychological symptoms of dementia. The literature search will be conducted based on search strategies designed for each database. The reviewers will independently assess the identified studies and extract the data. The risk of bias will be assessed and a meta-analysis will be conducted in accordance with the methodology for meta-analysis described in the Cochrane handbook. This systematic review will provide clinicians and policy makers with reliable evidence for developing and implementing nonpharmacological interventions for moderate to severe patients with dementia.
\end{abstract}

Psychiatry Investig 2018;15(4):417-423

Key Words Dementia, Activities of daily living, Behavioral and psychological symptoms of dementia.

\section{INTRODUCTION}

Dementia is a common neuropsychiatric syndrome that is usually chronic or progressive and causes deterioration of various mental functions, including cognitive, emotional, and mental. ${ }^{1}$ Dementia results in deterioration of the quality of life (QoL) of people with dementia (PWD) and their caregiv$\mathrm{ers}^{2}$ and the imposition of an enormous economic burden on the families of PWD and the public healthcare system., ${ }^{2,3}$ The global epidemic of dementia has caused pertinent global costs to rapidly increase. In 2010, the cost was 604 billion USD, and it is expected to reach 1 trillion USD by $2018 .{ }^{4}$

Unfortunately, a cure for dementia has not been found. Although pharmacological agents, such as acetylcholinesterase

\footnotetext{
Received: May 20, 2017 Revised: July 25, 2017

Accepted: September 6, 2017

$\triangle$ Correspondence: Ki Woong Kim, MD, PhD

Department of Neuropsychiatry, Seoul National University Bundang Hospital, 82 Gumi-ro 173beong-gil, Bundang-gu, Seongnam 13620, Republic of Korea Tel: +82-31-787-1821, Fax: +82-31-628-6848, E-mail: kwkimmd@snu.ac.kr

(a) This is an Open Access article distributed under the terms of the Creative Commons Attribution Non-Commercial License (http://creativecommons.org/licenses/bync/4.0) which permits unrestricted non-commercial use, distribution, and reproduction in any medium, provided the original work is properly cited.
}

inhibitors and N-methyl-D-aspartate receptor antagonists, are effective for temporary control of the symptoms of cognitive decline and improvement of the activities of daily living (ADL) of patients with Alzheimer's disease (AD), they cannot restore premorbid levels of function or maintain adequate levels of function in patients in later stages of dementia., ${ }^{5,6}$ Furthermore, medications, such as antipsychotics, should be sparingly used to manage the behavioral and psychological symptoms of dementia (BPSD) due to the risk of adverse events, including cerebrovascular events, tardive dyskinesia, neuroleptic malignant syndrome, agranulocytosis, and mortality.,8 Therefore, multifactorial therapeutic approaches that include pharmacological and nonpharmacological interventions (NPIVs) are increasingly advocated to improve the cognitive, affective, and global functioning of PWD, particularly for those in the later stages of dementia. ${ }^{7,-11}$

Recent systematic reviews have shown that several NPIVs may improve the cognition, communication, interactions, BPSD, ADL, and/or QoL of PWD. ${ }^{12-15}$ However, many of these reviews did not conduct meta-analyses due to lack of qualified studies and/or excessive study heterogeneity. ${ }^{16-23}$ Furthermore, most studies on NPIVs did not consider the severity of de- 
mentia or included PWD in the later stages, ${ }^{16-18,24-30}$ and the efficacy and/or mechanisms of NPIVs may differ in later stages of dementia compared to those in the earlier stages. ${ }^{7,8}$ Although three systematic reviews on the efficacy of NPIVs in people with moderate to severe dementia (PWMSD) have been conducted, the effect sizes of the NPIVs were not reported because the reviews were not meta-analyses. Furthermore, the times of publication or intervention settings of the studies included in the meta-analyses were limited. ${ }^{16,17,23,31,32}$

The principal strength of a systematic review is the capacity to identify salient and critical studies through the unmanageable numbers of existing medical literature using critical exploration, valuation, and synthesis. ${ }^{33}$ Second, the results of a systematic review could satisfy the need of decision makers for evidence-based integrated results. ${ }^{33}$ Conducting a systematic review, it is possible to refine the literature on the efficacy of NPIV in PWMSD by conducting qualitative synthesis and quantitative analysis, and also provide decision makers with reliable and pertinent evidence.

\section{METHODS}

\section{Purpose}

This systematic review, which will include a meta-analysis, aims to identify and evaluate the efficacy of NPIVs on the ADL and BPSD of PWMSD. Thus, the proposed systematic review will attempt to answer the following research questions:

1) Which NPIVs improve the ADL and BPSD of PWMSD?

2) What are the effects of NPIVs on the ADL and BPSD of PWMSD?

\section{Methodology}

This systematic review will be conducted in accordance with the Preferred Reporting Items for Systematic Reviews and Meta-Analyses (PRISMA) Statement ${ }^{34}$ and Cochrane Handbook for Systematic Reviews of Interventions. ${ }^{35}$ This systematic review protocol is registered with the PROSPERO (CRD42017058020). ${ }^{36}$

\section{Eligibility criteria}

\section{Populations}

The systematic review will include studies involving people diagnosed with any type of dementia according to the criteria in the Diagnostic and Statistical Manual of Mental Disorders, Third Edition (DSM-III); Fourth Edition, Text Revision (DSMIV-TR); or Fifth Edition (DSM-5); International Classification of Diseases, Tenth Revision (ICD-10); or other accepted diagnostic criteria.

This systematic review will include PWD in the moderate to severe stages who met one of the following criteria: Clinical Dementia Rating score of 2 or more, Global Deterioration Scale score of 5 or more, Functional Assessment Staging score of 5 or more, Mini-Mental State Examination (MMSE) score of 20 or less, or Revised Hasegawa's Dementia Scale score of 20 or less.

\section{Interventions}

This systematic review will include studies involving any type of NPIV that were conducted in community settings or institutional settings. NPIVs can be categorized in four broad groups following the practice guideline, ${ }^{8}$ and we will include several treatments such as cognitive therapy, art therapy, aromatherapy, massage, animal assisted therapy, exercise, or horticultural therapy.

\section{Outcomes}

This systematic review will employ ADL and BPSD as the primary outcomes and cognitive function and QoL as the secondary outcomes.

\section{Study design}

This systematic review will include randomized control trials (RCTs), quasi RCTs, non RCTs, cross-sectional studies, interrupted time series, and before-after studies that used the Study Design Algorithm for Medical Literature on Intervention. ${ }^{37}$

\section{Information sources}

This systematic review will search the following databases and reference lists of the included studies: Cochrane Central Register of Controlled Trials (CENTRAL), MEDLINE ${ }^{\circledR}$, EMBASE $^{\circledR}$, CINAHL, PsycINFO, KoreaMED, KMbase, and KISS. This systematic review will not limit the geography or time of the study, but only publications written in English or Korean will be included.

\section{Search strategy}

One reviewer $(\mathrm{R} \mathrm{Na})$ will search the information sources. The search strategy will include the study population and intervention terms suggested by Medical Subject Headings $\left(\mathrm{MeSH}^{\circledR}\right)$ and Emtree ${ }^{\circledR}$. The search terms will be adapted for use in other bibliographic databases with database-specific filters when available. Some syntax, including truncation, or Boolean operators, will be amended to the specific databases. A draft EMBASE search strategy is shown in Table 1. This EMBASE search strategy will be adapted to the syntax and subject headings of the other databases. 
Table 1. Example of an advanced search strategy-EMBASE

\begin{tabular}{|c|c|}
\hline 1 & 'dementia'/exp \\
\hline 2 & 'alzheimer disease'/exp \\
\hline 3 & 'frontotemporal dementia'/exp \\
\hline 4 & 'lewy body'/exp \\
\hline 5 & 'multiinfarct dementia'/exp \\
\hline 6 & 'normotensive hydrocephalus'/exp \\
\hline 7 & 'huntington chorea'/exp \\
\hline 8 & parkinson AND disease AND dementia \\
\hline 9 & vascular AND dementia \\
\hline 10 & alcohol AND related AND dementia \\
\hline 11 & $\begin{array}{l}\# 1 \text { OR \#2 OR \#3 OR \#4 OR \#5 OR \#6 OR \#7 OR \#8 OR } \\
\# 9 \text { OR \#10 }\end{array}$ \\
\hline 12 & moderate:ab,ti \\
\hline 13 & severe:ab,ti \\
\hline 14 & moderate AND to AND severe:ab,ti \\
\hline 15 & advanced:ab,ti \\
\hline 16 & profound:ab,ti \\
\hline 17 & \#12 OR \#13 OR \#14 OR \#15 OR \#16 \\
\hline 18 & \#11 AND \#17 \\
\hline 19 & 'therapy'/exp \\
\hline 20 & 'cognitive therapy'/exp \\
\hline 21 & 'art therapy'/exp \\
\hline 22 & 'aromatherapy'/exp \\
\hline 23 & 'massage'/exp \\
\hline 24 & 'touch'/exp \\
\hline 25 & 'animal assisted therapy'/exp \\
\hline 26 & 'exercise'/exp \\
\hline 27 & 'horticultural therapy'/exp \\
\hline 28 & 'virtual reality'/exp \\
\hline 29 & 'telerehabilitation'/exp \\
\hline 30 & $\begin{array}{l}\text { (nonpharmacological AND treatment\$) OR } \\
\text { (nonpharmacological AND therap\$) OR } \\
\text { (nonpharmacological AND intervention\$) }\end{array}$ \\
\hline 31 & $\begin{array}{l}\text { (nondrug AND treatment\$) OR (nondrug AND therap\$) } \\
\text { OR (nondrug AND intervention\$) }\end{array}$ \\
\hline 32 & emotion AND oriented AND intervention\$:ab,ti \\
\hline 33 & cognitive AND oriented AND intervention\$:ab,ti \\
\hline 34 & psychotherapy\$:ab,ti \\
\hline 35 & recreation AND therapy $\$: a b, t i$ \\
\hline 36 & validation AND therapy $\$: a b, t i$ \\
\hline 37 & reminiscence AND therapy $\$: a b, t i$ \\
\hline 38 & sensory AND stimulation AND intervention\$:ab,ti \\
\hline 39 & light AND therapy\$:ab,ti \\
\hline 40 & music AND therapy\$:ab,ti \\
\hline 41 & $\begin{array}{l}\text { (snoezelen:ab,ti) OR (snoezelen AND multisensory AND } \\
\text { stimulation:ab,ti) }\end{array}$ \\
\hline 42 & doll AND therapy\$:ab,ti \\
\hline
\end{tabular}

Table 1. Continued

\begin{tabular}{|c|c|}
\hline 43 & robot AND therapy\$:ab,ti \\
\hline 44 & multimodal AND therapy\$:ab,ti \\
\hline 45 & occupational AND therapy\$:ab,ti \\
\hline 46 & behavi?r AND therapy $\$: a b, t i$ \\
\hline 47 & computer AND assisted AND therapy\$:ab,ti \\
\hline 48 & reality AND orientation $\$: a b, t i$ \\
\hline 49 & cognitive AND training $\$$ :ab,ti \\
\hline \multirow[t]{5}{*}{50} & $\# 19$ OR \#20 OR \#21 OR \#22 OR \#23 OR \#24 OR \#25 OR \\
\hline & $\# 26 \mathrm{OR} \# 27 \mathrm{OR} \# 28$ OR \#29 OR \#30 OR \#31 OR \#32 OR \\
\hline & \#33 OR \#34 OR \#35 OR \#36 OR \#37 OR \#38 OR \#39 OR \\
\hline & $\# 40$ OR \#41 OR \#42 OR \#43 OR \#44 OR \#45 OR \#46 OR \\
\hline & \#47 OR \#48 OR \#49 \\
\hline 51 & \#18 AND \#50 \\
\hline 52 & 'randomized controlled trial'/exp \\
\hline 53 & 'multicenter study'/exp \\
\hline 54 & 'clinical trial'/exp \\
\hline 55 & 'randomization'/exp \\
\hline 56 & 'single blind procedure'/exp \\
\hline 57 & 'crossover procedure'/exp \\
\hline 58 & 'placebo'/exp \\
\hline 59 & 'prospective study'/exp \\
\hline 60 & double AND blind AND procedure \\
\hline 61 & randomi?ed AND controlled AND trial\$ \\
\hline 62 & rct:ab,ti \\
\hline 63 & random AND allocation \\
\hline 64 & randomly AND allocated \\
\hline 65 & allocated AND randomly \\
\hline 66 & single AND blind\$:ab,ti \\
\hline 67 & double AND blind\$:ab,ti \\
\hline 68 & cross AND sectional AND study \\
\hline 69 & before AND after AND study \\
\hline 70 & \#52 OR \#53 OR \#54 OR \#55 OR \#56 OR \#57 OR \#58 OR \\
\hline & \#59 OR \#60 OR \#61 OR \#62 OR \#63 OR \#64 OR \#65 OR \\
\hline & \#66 OR \#67 OR \#68 OR \#69 \\
\hline 71 & 'retrospective study'/exp \\
\hline 72 & 'cohort'/exp \\
\hline 73 & 'letter'/exp \\
\hline 74 & case AND report \\
\hline 75 & case AND study \\
\hline 76 & abstract AND report \\
\hline 77 & $\# 71$ OR \#72 OR \#73 OR \#74 OR \#75 OR \#76 \\
\hline 78 & \#70 NOT \#77 \\
\hline 79 & \#51 AND \#78 \\
\hline 80 & animal \\
\hline 81 & human \\
\hline 82 & \#80 NOT (\#80 AND \#81) \\
\hline 83 & \#79 NOT \#82 \\
\hline
\end{tabular}




\section{Study records}

\section{Data management}

This systematic review will export the search results to EndNote $^{\mathrm{TM}}$ X8.0.1 [Clarivate Analytics (formerly Thomson Reuters), Philadelphia, PA, USA] in which all reference records will be managed.

\section{Selection process}

Two reviewers ( $\mathrm{R} \mathrm{Na}$ and YJ Kim) will independently select studies in the three phases listed below. If any discrepancies occur between the two reviewers, two additional reviewers (KW Kim and K Kim) will amend the decision. This systematic review will create a PRISMA flow diagram of the included and excluded studies.

1) Initial screening: potential papers will be identified for abstract retrieval, and any obviously irrelevant studies will be eliminated by screening their titles.

2) Secondary screening: potential papers will be identified for full text retrieval by screening their abstracts.

3) Tertiary screening: papers that should be included in the current systematic review will be identified by reading the full text, and the reasons for exclusion will be documented.

\section{Data collection process}

Two reviewers (R Na and YJ Kim) will collect the data. Eli- gible data will be independently extracted with a standardized form. Any discrepancies will be resolved by deliberation between $\mathrm{R} \mathrm{Na}$ and YJ Kim or, if needed, with two other reviewers (KW Kim and K Kim).

\section{Data items}

Two reviewers ( $\mathrm{R} \mathrm{Na}$ and YJ Kim) will extract the data from the publications with a standardized data extraction form. The extracted data will be summarized as shown in Table 2.

\section{Outcomes and prioritization}

\section{Primary outcomes}

The primary outcomes of this systematic review will be ADL and BPSD of PWMSD. ADL, which will include Basic and Instrumental ADL, ${ }^{38}$ will be measured with standardized assessment scales, including the AD Cooperative Study's ADL scale, Barthel Index, Disability Assessment for Dementia, DScale of Change, Katz Index, modified instrumental ADL scale, modified Physical Self-Maintenance Scale, Functional Independence Measure, and Nurse Informant Index of Activities of Daily Living. ${ }^{12,38-43}$

The other primary outcome, BPSD, represents a heterogeneous group of noncognitive symptoms and behaviors. ${ }^{44}$ BPSD includes agitation, aggression, aberrant motor behavior, anxiety, elation, irritability, depression, dysphoria, apathy, dis-

Table 2. Data extraction variables

\begin{tabular}{|c|c|}
\hline Content & Data items \\
\hline Prospective study information & Author(s), Year of publication, Country \\
\hline Study design & Trial design \\
\hline Number of participants & $\begin{array}{l}\text { Number of participants invited, number of participants screened, number of participants eligible, } \\
\text { number of participants randomized, reasons for non-eligibility, reasons for drop-out }\end{array}$ \\
\hline Population & $\begin{array}{l}\text { Severity of dementia, Type of dementia (e.g. Alzheimer's disease, vascular dementia, dementia with } \\
\text { Lewy bodies, frontotemporal dementia, etc.) Average age, Gender, ethnicity, Diagnostic criteria for } \\
\text { dementia (e.g. DSM-III, DSM-IV, DSM-5, ICD-10, etc.), Diagnostic tool for severity of dementia } \\
\text { (e.g. CDR, GDS, FAST, MMSE, etc.), Physical health comorbidity, Usage of antidementia drug }\end{array}$ \\
\hline Intervention & $\begin{array}{l}\text { Name of treatment group, Type of non-pharmacological intervention, Frequency and duration } \\
\text { of intervention, Intervention provider, length of intervention session, Mode of delivery } \\
\text { (e.g. individual, group, combination) }\end{array}$ \\
\hline Comparison & $\begin{array}{l}\text { Name and type of control group, Frequency and duration of intervention, Intervention provider, } \\
\text { length of intervention session, Mode of delivery (e.g. individual, group, combination) }\end{array}$ \\
\hline Outcome & Detail of outcome measure \\
\hline Outcome measure & Baseline, Follow-up assessment, Losses to follow-up, Result \\
\hline Follow-up & Duration of follow-up, Drop-out rate \\
\hline Setting & Community, Institution \\
\hline
\end{tabular}


inhibition, delusions, hallucinations, appetite or eating changes, euphoria, sleep and night-time behavior changes, vegetative features, fear, phobia, wandering, threat, violence, fearfulness, diurnal rhythm disturbances, psychosis, and mood changes. ${ }^{44-47}$ BPSD assessments require standardized clinical assessments, such as the Behavioral Pathology in AD Scale, Neuropsychiatric Inventory, Behavior Rating Scale for Dementia, Cornell Scale for Depression in Dementia, Geriatric Depression Scale, Behavior and Mood Disturbance Scale, Rehabilitation Evaluation of Hall and Baker, Clifton Assessment Procedures for the Elderly, Cohen-Mansfield Agitation Inventory, and Actigraphy. ${ }^{31,44,48-51}$

\section{Secondary outcomes}

The secondary outcomes will include the cognitive function and QoL of PWD. Cognitive function will be measured with standardized assessment tools, such as the Mini-Mental State Examination (MMSE), Severe Impairment Battery, Kingston Dementia Rating Scale, AD Assessment Scale-Cognitive Subscale, Brief Cognitive Screening Battery, Consortium to Establish a Registry for $\mathrm{AD}$ Assessment Tool (Korean version), Modified MMSE, and Montreal Cognitive Assessment. ${ }^{12,32,52-54}$ The evaluations of the QoL variables in PWD will require standardized and validated QoL scales, such as the Quality of Life scale in AD, AD Related Quality of Life, Dementia-Quality of Life, EuroQoL-Five Dimensions Questionnaire, and Blau's QoL Scale. ${ }^{14,55-59}$

\section{Risk of bias in individual studies}

Two reviewers ( $\mathrm{R} \mathrm{Na}$ and YJ Kim) will independently assess the risk of bias (RoB) in each study. All reviewers will discuss the quality of a study if the two reviewers disagree. The RoB of RCTs will be assessed with the RoB scale, ${ }^{60}$ while the RoB of nonRCTs will be assessed with the RoB Assessment Tool for Nonrandomized Studies (RoBANS). ${ }^{61}$ Each included study will be classified as low risk, high risk, or unclear according to the RoB and/or RoBANS.

\section{Data synthesis}

Two reviewers (YJ Kim and R Na) will conduct a meta-analysis of the outcomes with Review Manager if a sufficient number of studies are included. They will employ risk and odds ratios with 95\% confidence intervals (CIs) for dichotomous outcomes and weighted mean differences (with 95\% CIs) or standardized mean differences (with 95\% CIs) for continuous outcomes. For missing data, we will attempt to contact the authors of the study to obtain the relevant information. Intention-to-treat data, if available, will be preferred.

This systematic review will consider the various characteristics of the participants and the study designs when evaluat- ing the heterogeneity of the studies. Heterogeneity will be statistically tested with the chi-squared and I-squared tests. ${ }^{35}$ To address heterogeneity, a meta-analysis will be conducted by considering the following: re-examining the data for accuracy, canceling the meta-analysis, exploring the heterogeneity, ignoring the heterogeneity, performing a random-effects metaanalysis, changing the effect measure, and/or excluding studies. ${ }^{35}$

This systematic review will conduct subgroup analyses of the participants (e.g., dementia subtype, dementia severity, or participant characteristics), interventions (types, providers, or settings), study designs, and follow-up periods if sufficient data are available.

\section{Meta-biases}

This systematic review will assess publication bias with funnel plots of potential reporting bias if the number of studies in the meta-analysis is over 10 .

\section{Confidence in the cumulative estimates}

This systematic review will assess the quality of the evidence for all outcomes with the Grading of Recommendations Assessment, Development and Evaluation (GRADE) ${ }^{62}$ All reviewers will determine the quality of the evidence according to the GRADE guidelines. ${ }^{62-70}$

\section{CONCLUSION}

This systematic review will examine the clinical efficacy of NPIVs on the ADL, BPSD, cognitive function, and QoL of PWMSD. Although the efficacy of NPIVs on PWD may differ according to the severity of dementia, recent reviews on NPIVs for PWD have not separately evaluated NPIVs efficacy on PWMSD or conducted relevant meta-analyses. To maximize the power of systematic reviews, restriction of publication language is not recommended. However, this systematic review will include studies written in English or Korean only for practical reasons, which will be a limitation of this study.

Nonetheless, this systematic review will provide clinicians and policy makers with reliable evidence for developing and implementing NPIVs for PWMSD.

\section{Acknowledgments}

This systematic review is supported by a grant from the National Institute of Dementia of Korea (Grant Number: NIDR-1703-0018), Republic of Korea.

\section{REFERENCES}

1. Organization WH. The ICD-10 Classification of Mental and Behavioural Disorders: Clinical Descriptions and Diagnostic Guidelines. Geneva: World Health Organization; 1992.

2. Cho H, Ko Z. Current state of senile dementia and improvement of the long term care insurance for elderly people. J Kor Acad Industr 
Coop Soc 2012;13:5816-5825.

3. Organization WH. First WHO Ministerial Conference on Global Action against Dementia: Meeting Report, WHO Headquarters, Geneva, Switzerland, 16-17 March, 2015.

4. Prince MJ. World Alzheimer Report 2015: the Global Impact of Dementia: An Analysis of Prevalence, Incidence, Cost and Trends. London: Alzheimer's Disease International; 2015.

5. Gardette V, Coley N, Andrieu S. Non-pharmacologic therapies: a different approach to ad. Can Rev of Alz Dis Other Dem 2010;13:13-22.

6. O'Hara R, Mumenthaler MS, Yesavage JA. Update on Alzheimer's disease: recent findings and treatments. West J Med 2000;172:115-120.

7. Committee GA. Clinical Practice Guidelines and Principles of Care for People with Dementia. Sydney: Guidelines Adaptation Committee; 2016.

8. Rabins PV, Blacker D, Rovner BW, Rummans TA, Schneider LS, Tariot PN, et al. American Psychiatric Association practice guideline for the treatment of patients with Alzheimer's disease and other dementias. Am J Psychiatry 2007;164(12 Suppl):5-56.

9. Han JW, Oh K, Yoo S, Kim E, Ahn KH, Son YJ, et al. Development of the ubiquitous spaced retrieval-based memory advancement and rehabilitation training program. Psychiatry Investig 2014;11:52-58.

10. Jean L, Bergeron ME, Thivierge S, Simard M. Cognitive intervention programs for individuals with mild cognitive impairment: systematic review of the literature. Am J Geriatr Psychiatry 2010;18:281-296.

11. Yesavage JA, Westphal J, Rush L. Senile dementia: combined pharmacologic and psychologic treatment. J Am Geriatr Soc 1981;29:164-171.

12. Forbes D, Blake CM, Thiessen EJ, Peacock S, Hawranik P. Light therapy for improving cognition, activities of daily living, sleep, challenging behaviour, and psychiatric disturbances in dementia. Cochrane Database Syst Rev 2014;(2):CD003946.

13. Forrester LT, Maayan N, Orrell M, Spector AE, Buchan LD, Soares Weiser K. Aromatherapy for dementia. Cochrane Database Syst Rev 2014;(2):CD003150.

14. Orgeta V, Qazi A, Spector AE, Orrell M. Psychological treatments for depression and anxiety in dementia and mild cognitive impairment. Cochrane Database Syst Rev 2014;(1):CD009125.

15. Woods B, Aguirre E, Spector AE, Orrell M. Cognitive stimulation to improve cognitive functioning in people with dementia. Cochrane Database Syst Rev 2012;(2):CD005562.

16. de Oliveira AM, Radanovic M, de Mello PC, Buchain PC, Vizzotto $\mathrm{AD}$, Celestino DL, et al. Nonpharmacological interventions to reduce behavioral and psychological symptoms of dementia: a systematic review. Biomed Res Int 2015;2015:218980. doi: 10.1155/2015/218980.

17. Brodaty H, Arasaratnam C. Meta-analysis of nonpharmacological interventions for neuropsychiatric symptoms of dementia. Am J Psychiatry 2012;169:946-953.

18. Littbrand H, Stenvall M, Rosendahl E. Applicability and effects of physical exercise on physical and cognitive functions and activities of daily living among people with dementia: a systematic review. Am J Phys Med Rehabil 2011;90:495-518.

19. Hermans D, Htay U, Cooley SJ. Non pharmacological interventions for wandering of people with dementia in the domestic setting. Cochrane Database Syst Rev 2007;(1):CD005994.

20. Hansen NV, Jørgensen T, Ørtenblad L. Massage and touch for dementia. Cochrane Database Syst Rev 2006;(4):CD004989.

21. Neal M, Briggs M. Validation therapy for dementia. Cochrane Database Syst Rev 2000;(2):CD001394.

22. Vink AC, Bruinsma MS, Bruinsma MS, Scholten RJ. Music therapy for people with dementia. Cochrane Database Syst Rev 2004;(3):CD003477.

23. Chung JC, Lai CK, Chung PM, French HP. Snoezelen for dementia. Cochrane Database Syst Rev 2002;(4):CD003152.

24. Brasure M, Jutkowitz E, Fuchs E, Nelson VA, Kane RA, Shippee T, et al. Nonpharmacologic Interventions for Agitation and Aggression in Dementia. Rockville, MD: Agency for Healthcare Research and Quality (US); 2016. Report No.: 16-EHC019-EF.
25. Spector A, Orrell M, Lattimer M, Hoe J, King M, Harwood K, et al. Cognitive behavioural therapy (CBT) for anxiety in people with dementia: study protocol for a randomised controlled trial. Trials 2012; 13:197.

26. Buschert VC, Friese U, Teipel SJ, Schneider P, Merensky W, Rujescu D, et al. Effects of a newly developed cognitive intervention in amnestic mild cognitive impairment and mild Alzheimer's disease: a pilot study. J Alzheimers Dis 2011;25:679-694.

27. O’Neil ME, Freeman M, Christensen V, Telerant R, Addleman A, Kansagara D. A systematic evidence review of non-pharmacological interventions for behavioral symptoms of dementia. Washington, DC: Department of Veterans Affairs; 2011.

28. Bottino CM, Carvalho IA, Alvarez AMM, Avila R, Zukauskas PR, Bustamante SE, et al. Cognitive rehabilitation combined with drug treatment in Alzheimer's disease patients: a pilot study. Clin Rehabil 2005;19:861-869.

29. Burns A, Guthrie E, Marino-Francis F, Busby C, Morris J, Russell E, et al. Brief psychotherapy in Alzheimer's disease. Br J Psychiatry 2005; 187:143-147.

30. Chapman SB, Weiner MF, Rackley A, Hynan LS, Zientz J. Effects of cognitive-communication stimulation for Alzheimer's disease patients treated with donepezil. J Speech Lang Hear Res 2004;47:1149-1163.

31. Kverno KS, Black BS, Nolan MT, Rabins PV. Research on treating neuropsychiatric symptoms of advanced dementia with non-pharmacological strategies, 1998-2008: a systematic literature review. Int Psychogeriatr 2009;21:825-843.

32. Boote J, Lewin V, Beverley C, Bates J. Psychosocial interventions for people with moderate to severe dementia: a systematic review. Clin Effect Nurs 2006;9(Suppl 1):e1-e15.

33. Mulrow CD. Rationale for systematic reviews. BMJ 1994;309:597-599.

34. Shamseer L, Moher D, Clarke M, Ghersi D, Liberati A, Petticrew M, et al. Preferred reporting items for systematic review and meta-analysis protocols (PRISMA-P) 2015: elaboration and explanation. BMJ 2015; 349:g7647.

35. Higgins JP, Green S. Cochrane Handbook for Systematic Reviews of Interventions. West Sussex: John Wiley \& Sons; 2011.

36. Kim KW, Kim K, Kim YJ, Na R. A systematic review of non-pharmacological interventions for moderate to severe dementia. PROSPERO 2017 CRD42017058020 Available from: http://www.crd.york.ac.uk/ PROSPERO/display_record.php?ID=CRD42017058020.

37. Seo HJ, Kim SY, Lee YJ, Jang BH, Park JE, Sheen SS, et al. A newly developed tool for classifying study designs in systematic reviews of interventions and exposures showed substantial reliability and validity. J Clin Epidemiol 2016;70:200-205.

38. Buzaid A, Dodge MP, Handmacher L, Kiltz PJ. Activities of daily living: evaluation and treatment in persons with multiple sclerosis. Phys Med Rehabil Clin N Am 2013;24:629-638.

39. Hartigan I. A comparative review of the Katz ADL and the Barthel Index in assessing the activities of daily living of older people. Int J Older People Nurs 2007;2:204-212.

40. Aisen PS, Schafer KA, Grundman M, Pfeiffer E, Sano M, Davis KL, et al. Effects of rofecoxib or naproxen vs placebo on Alzheimer disease progression: a randomized controlled trial. JAMA 2003;289:28192826.

41. Feldman H, Gauthier S, Hecker J, Vellas B, Emir B, Mastey V, et al. Efficacy of donepezil on maintenance of activities of daily living in patients with moderate to severe Alzheimer's disease and the effect on caregiver burden. J Am Geriatr Soc 2003;51:737-744.

42. Rüther E, Glaser A, Bleich S, Degner D, Wiltfang J. A prospective PMS study to validate the sensitivity for change of the $\mathrm{D}$-scale in advanced stages of dementia using the NMDA-antagonist memantine. Pharmacopsychiatry 2000;33:103-108.

43. Gélinas I, Gauthier L, McIntyre M, Gauthier S. Development of a functional measure for persons with Alzheimer's disease: the disability assessment for dementia. Am J Occup Ther 1999;53:471-481. 
44. Cerejeira J, Lagarto L, Mukaetova-Ladinska E. Behavioral and psychological symptoms of dementia. Front Neurol 2012;3:73.

45. Pilotto A, Ferrucci L, Franceschi M, D’Ambrosio LP, Scarcelli C, Cascavilla L, et al. Development and validation of a multidimensional prognostic index for one-year mortality from comprehensive geriatric assessment in hospitalized older patients. Rejuvenation Res 2008;11: 151-161.

46. Jacobs MR, Strauss ME, Patterson MB, Mack JL. Characterization of depression in Alzheimer's disease by the CERAD Behavior Rating Scale for Dementia (BRSD). Am J Geriatr Psychiatry 1998;6:53-58.

47. Reisberg B, Auer SR, Monteiro IM. Behavioral pathology in Alzheimer's disease (BEHAVE-AD) rating scale. Int Psychogeriatr 1996;8(Suppl 3):301-308.

48. Kørner A, Lauritzen L, Abelskov K, Gulmann N, Marie Brodersen A, Wedervang-Jensen $\mathrm{T}$, et al. The geriatric depression scale and the cornell scale for depression in dementia. A validity study. Nord J Psychiatry 2006;60:360-364.

49. Tariot PN, Mack JL, Patterson MB, Edland SD, Weiner MF, Fillenbaum $\mathrm{G}$, et al. The behavior rating scale for dementia of the consortium to establish a registry for Alzheimer's disease. Am J Psychiatry 1995;152: 1349-1357.

50. Cummings JL, Mega M, Gray K, Rosenberg-Thompson S, Carusi DA, Gornbein J. The neuropsychiatric inventory comprehensive assessment of psychopathology in dementia. Neurology 1994;44:2308-2314.

51. Alexopoulos GS, Abrams RC, Young RC, Shamoian CA. Cornell scale for depression in dementia. Biol Psychiatry 1988;23:271-284.

52. Sink KM, Craft S, Smith SC, Maldjian JA, Bowden DW, Xu J, et al. Montreal cognitive assessment and modified mini mental state examination in African Americans. J Aging Res 2015;2015:872018. doi:10.1155/2015/872018.

53. Doraiswamy PM, Kaiser L, Bieber F, Garman RL. The Alzheimer's Disease Assessment Scale: evaluation of psychometric properties and patterns of cognitive decline in multicenter clinical trials of mild to moderate Alzheimer's disease. Alzheimer Dis Assoc Disord 2001;15:174-183.

54. Panisset M, Roudier M, Saxton J, Boiler F. Severe impairment battery. A neuropsychological test for severely demented patients. Arch Neurol 1994;51:41-45.

55. González Salvador T, Lyketsos CG, Baker A, Hovanec L, Roques C, Brandt J, et al. Quality of life in dementia patients in long term care. Int J Geriatr Psychiatry 2000;15:181-189.

56. Hoe J, Katona C, Roch B, Livingston G. Use of the QOL-AD for measuring quality of life in people with severe dementia-the LASER-AD study. Age Ageing 2005;34:130-135.

57. Ankri J, Beaufils B, Novella JL, Morrone I, Guillemin F, Jolly D, et al. Use of the EQ-5D among patients suffering from dementia. J Clin Epidemiol 2003;56:1055-1063.

58. Rabins P. Measuring quality of life in persons with dementia. Int Psychogeriatr 2000;12(Supp 1):47-49.

59. Brod M, Stewart AL, Sands L, Walton P. Conceptualization and measurement of quality of life in dementia: the dementia quality of life instrument (DQoL). Gerontologist 1999;39:25-36.

60. Higgins JP, Altman DG, Gøtzsche PC, Jüni P, Moher D, Oxman AD, et al. The Cochrane Collaboration's tool for assessing risk of bias in randomised trials. BMJ 2011;343:d5928.

61. Kim SY, Park JE, Lee YJ, Seo HJ, Sheen SS, Hahn S, et al. Testing a tool for assessing the risk of bias for nonrandomized studies showed moderate reliability and promising validity. J Clin Epidemiol 2013;66:408414.

62. Guyatt G, Oxman AD, Akl EA, Kunz R, Vist G, Brozek J, et al. GRADE guidelines: 1. Introduction GRADE evidence profiles and summary of findings tables. J Clin Epidemiol 2011;64:383-394.

63. Guyatt GH, Oxman AD, Kunz R, Atkins D, Brozek J, Vist G, et al. GRADE guidelines: 2. Framing the question and deciding on important outcomes. J Clin Epidemiol 2011;64:395-400.

64. Balshem H, Helfand M, Schünemann HJ, Oxman AD, Kunz R, Brozek J, et al. GRADE guidelines: 3. Rating the quality of evidence. J Clin Epidemiol 2011;64:401-406.

65. Guyatt GH, Oxman AD, Vist G, Kunz R, Brozek J, Alonso-Coello P, et al. GRADE guidelines: 4 . Rating the quality of evidence-study limitations (risk of bias). J Clin Epidemiol 2011;64:407-415.

66. Guyatt GH, Oxman AD, Montori V, Vist G, Kunz R, Brozek J, et al. GRADE guidelines: 5 . Rating the quality of evidence-publication bias. J Clin Epidemiol 2011;64:1277-1282.

67. Guyatt GH, Oxman AD, Kunz R, Brozek J, Alonso-Coello P, Rind D, et al. GRADE guidelines 6 . Rating the quality of evidence-imprecision. J Clin Epidemiol 2011;64:1283-1293.

68. Guyatt GH, Oxman AD, Kunz R, Woodcock J, Brozek J, Helfand M, et al. GRADE guidelines: 7. Rating the quality of evidence-inconsistency. J Clin Epidemiol 2011;64:1294-1302.

69. Guyatt GH, Oxman AD, Kunz R, Woodcock J, Brozek J, Helfand M, et al. GRADE guidelines: 8 . Rating the quality of evidence-indirectness. J Clin Epidemiol 2011;64:1303-1310.

70. Guyatt GH, Oxman AD, Sultan S, Glasziou P, Akl EA, Alonso-Coello P, et al. GRADE guidelines: 9. Rating up the quality of evidence. J Clin Epidemiol 2011;64:1311-1316. 